\title{
Exploring Multi-Objective Trade-Offs in the Design Space of a Waste Heat Recovery System
}

\author{
Maizura Mokhtar \\ School of Engineering \& the Built Environment, Edinburgh Napier University, Merchiston \\ Campus, Edinburgh, EH10 5DT, UK \\ Stephen Burns \\ SRUC Research Division, Scotland's Rural College (SRUC), Roslin Institute Building, \\ Easter Bush, Midlothian, EH25 9RG, UK \\ Dave Ross \\ SRUC Research Division, Scotland's Rural College (SRUC), Roslin Institute Building, \\ Easter Bush, Midlothian, EH25 9RG, UK \\ Ian Hunt \\ School of Engineering \&5 the Built Environment, Edinburgh Napier University, Merchiston \\ Campus, Edinburgh, EH10 5DT, UK
}

\begin{abstract}
A waste heat recovery system (WHRS) is used to capture waste heat released from an industrial process, and transform the heat into reusable energy. In practice, it can be difficult to identify the optimal form of a WHRS for a particular installation, since this can depend on various design objectives, which are often mutually exclusive. More so when the number of objectives is large. To address this problem, a multiobjective evolutionary algorithm (MOEA) was used to explore and characterise the trade-off surface within the design space of a particular WHRS. A combination of clustering algorithm and parallel coordinates plots was proposed for use in analysing the results. The trade-off surface is first segmented using a clustering algorithm and parallel coordinates plots are then used to both visualise and understand the resulting set of Pareto-optimal
\end{abstract}

\footnotetext{
${ }^{*}$ Corresponding author

Email address: M.Mokhtar@napier.ac.uk (Maizura Mokhtar)
}

Preprint submitted to Applied Energy

February 20, 2017 
designs. As a case study, a simulation of a WHRS commonly found in the food and drinks process industries was developed, comprising of a desuperheater coupled to a hot water reservoir. The system was parameterised, considering typical objectives, and the MOEA used to build a library of alternative Pareto-optimal designs that can be used by installers. The resulting visualisation are used to better understand the sensitivity of the system's parameters and their trade-offs, providing another source of information for prospective installations.

Keywords: waste heat recovery, optimisation, multi-objective evolutionary algorithm, mutually exclusive objective functions

\section{Nomenclature}

$n$ number of objectives

$u$ number of evolved parameters

$k$ number of clusters

$\dot{m}_{w d}$ mass flow rate of water into the $\mathrm{DSH} / \mathrm{HWR}$

$\dot{m}_{w d_{\max }}$ maximum mass flow rate of water into the DSH/HWR

$m_{w t}$ mass of water in the HWR

$m_{w t_{\min }}$ minimum mass of water in the HWR

$m_{w t_{\max }}$ maximum mass of water in the HWR

$P_{b}$ power of the backup heater

$P_{b_{\max }}$ maximum power of the backup heater

$T_{h w}$ required/demanded hot water temperature

$T_{m x}$ maximum water temperature in the HWR

$T_{m}$ mains water temperature

$T_{r i}$ input refrigerant temperature to the DSH 
$T_{r o}$ output refrigerant temperature from the DSH

$T_{w i}$ input water temperature to the DSH

$T_{w o}$ output water temperature from the DSH

$T_{w t}$ water temperature in the HWR

$\Delta T_{\max }$ maximum difference between $T_{m x}$ and $T_{h w}$

DSH Desuperheater

EA Evolutionary algorithm

HWR Hot water reservoir

MOEA Multiobjective evolutionary algorithm

MOOA Multiobjective optimisation algorithm

WHRS Waste heat recovery system

\section{Introduction}

Many energy systems have behaviours that are sensitive to their parameter values, and these values need to be optimised [1, 2]. Many of these systems also have multiple objectives, and these are often conflicting, meaning that in practice there is not a single design that satisfies every objective. In this situation, choosing an appropriate set of parameters first involves understanding the different trade-offs that can be made within the system's design space. One way of doing this is to identify all the solutions that are no better or worse than each other when considered across all objectives. This is known as the Pareto optimal set. Multi-objective optimisation algorithms (MOOA) are a group of optimisation techniques that are able to find good approximations of the Pareto optimal set. Because of this, they have become increasingly popular in the design of engineering systems [3], [4, [5, [6], including the optimisation 
of energy systems. Examples are described in [7, [8, 9], 10, 11], 12, [13, [14, [15, [16], [17.

A Waste Heat Recovery System (WHRS) captures waste heat released from an industrial process, storing it in a form that can later be reused, for example using a hot water reservoir (HWR). A WHRS is a good example of a system with mutually conflicting objectives. Common practise in WHRS optimisation involves optimising using conventional single objective methods, typically Mixed Integer (Non-)Linear Programming (MILP or MINLP) [18, [19, 20], 21], 22, [23. In cases where there are multiple objectives, these are scalarised into a single objective. Alternatively, optimisation can be done using only a single objective, most typically minimising the cost of the installation and operations summed together, and the remaining objectives are implemented as constraints [18, 19], 21], 22], 23]. Given that single objective optimisation only provides a single solution, any information on objective trade-offs will be lost during the optimisation process, and any analysis of how the parameters affect the system efficiency will also be lost. In this work, by comparison, a Multi-Objective Evolutionary Algorithm (MOEA) was used to carry out multi-objective optimisation of a WHRS, using the results to visualise trade-offs in the design space.

As a case study, a simulation of a type of WHRS commonly found in the food and drinks process industry was developed, which involves a desuperheater connected to a HWR, providing hot water for a production plant's intermittent internal cleaning process. After identifying a number of common objectives for this type of system, the MOEA was used to find a set of trade-off designs within this objective space.

One disadvantage of MOOA and MOEA approaches is the visualisation of results, especially when $n$ number of objectives and $u$ number of parameters are large. A common method of visualising the results is to use a scatter plot, or decision maps, as shown in [14, [10], 13], but in general these can only show three objectives or parameters at once, which is limiting. Furthermore, the correlation between parameter and objective values are not explicitly shown, and in practice, analysis of the correlation is only applied to a selected few 
solutions, as depicted in [7, 8], 9]. Typically, they are the solutions at the extrema of the Pareto-optimal set, giving limited insight to the attributes of the other Pareto-optimal solutions found. Consequently many studies limit themselves to $n \leq 3$ objectives, as shown in [9], 13, [11, 17, 8].

To aid in the analysis of the results from high-dimensional multi-objective optimisation, and to identify a reduced set of representative designs, this paper proposed an alternative method of visualising the Pareto-optimal solutions. The solutions are first clustered into $k$-number of clusters, either in the design space or the parameter space, to identify the degree of commonality between the solutions. For each cluster identified, parallel coordinates [24, 25] are used to visualise the high-dimensional solution space and objective space as a pair of two-dimensional plots; one for each of the spaces.

Parallel coordinate plots are used to visualise the Pareto-optimal solutions, each for the $u$-dimensional solution space and $n$-dimensional objective space. The correlation between a solution and its objective values in a specific cluster are identified by the common colour used in both plots. This method of visualisation can therefore reduce the number of figures (and tables) to depict the results significantly, down to $2 k$ figures - one for each of the two spaces. The significant reduction in the number of figures used eases in the analysis of the trade-offs between the Pareto-optimal solutions.

The paper is organised as follows: Section 2 provides a brief introduction to the MOOA used in this work. Section 3 introduces WHRS and gives an overview of the case study. Section 4 presents results and analysis using various multidimensional visualisation methods, including decision maps and parallel coordinates plots. Section 5 concludes the paper.

\section{Multi-Objective Evolutionary Algorithm (MOEA)}

Whilst many forms of optimisation can be generalised to the multi-objective case, in practice the most widely used forms of MOOA are based around evolutionary algorithms (EA). EAs are a class of population-based metaheuristic 
optimisation algorithms. As described in [26]: the initial population is a random sample of search points, a selection mechanism then discards search points with poor objective values, and variation operators derive a new population of search points from those that remain. This new population then replaces the previous population, and the process of selection and variation are repeated until an optimal solution is found, or some other termination criterion is met. The search points, in our case, are vectors of parameter values. The variation operators are crossover, which recombines two search points by swapping vector elements, and mutation, which randomly replaces one or more vector elements to create a new search point. Population-based metaheuristics, such as EAs, carry out a relatively broad search of an optimisation space, and consequently are often able to find better solutions than local search metaheuristics, such as hill climbing or simulated annealing.

A multi-objective evolutionary algorithm (MOEA) is a specialised form of EA, and in this work, a popular MOEA called NSGA-II [27] was used. The main difference between NSGA-II and a single-objective EA lies in how selection takes place. Rather than only propagating the best search points from one generation to the next, NSGA-II first carries out a ranking of the search points in the population. Search points which are no worse than any other when considered across all the objectives are known as dominating search points, and are given a rank of 1 . Those which are only dominated by rank 1 search points are assigned rank 2, etc. After ranking, the first half of the ordered population is then copied directly to the next generation, and the remainder of the population is filled by applying the variation operators.

NSGA-II also uses a diversity preservation method, known as crowding, to encourage search in regions of objective space which have not been previously explored. For the search points that are of the same rank, those that are more dissimilar to the others are preferred for selection for the next generation population. This not only results in an approximation of the Pareto optimal set which has a good spread of solutions across the objective space, but also helps to discourage convergence to local optima. 
In addition to its wide use, not limited to the works described in [17, 11, [15], 14], and reviews described in 28], 29, NSGA-II was also chosen because reviews conducted by [12] indicated that NGSA-II outperformed other MOEAs (ESPEA, NSGA-III and SPEA2) when optimising a smart building's energy storage system, a system with notable similarities to a WHRS [30]. The authors of [12] use, among other metrics, hypervolume (Section 4.1] to measure the performance of the algorithms. They summarised that NGSA-II (together with ESPEA, developed by one of the authors of [12] in 2015) achieved the largest hypervolumes. The larger the hypervolume, the better the optimiser. Given the maturity of NSGA-II against ESPEA, NSGA-II was chosen to optimise the WHRS.

\section{Waste Heat Recovery System (WHRS)}

Various factors have motivated an increase in WHRS installations within the process industries. One prominent factor is the need to save on energy costs, which have increased markedly in recent years. Another is environmental regulations, reflecting the growing need to reduce waste heat released to the environment 31], 32, for example the commitment reached at the 2015 UN Climate Change Conference in Paris, France, to limit the global temperature rise to $2^{\circ} \mathrm{C}$. In the case of the UK, it is also driven by the Climate Change Act [33, which stipulates that carbon gas emissions should be reduced by at least $80 \%$ from 1990 levels by 2050 .

This work focuses on the food and drinks process industries, which create large volumes of waste heat whilst at the same time requiring large volumes of heat within their processes. Waste heat is generated from processes such as refrigeration, from cleaning, and from improper insulation of the plant. Heat is required, for instance, to produce the hot water needed for food preparation and the cleaning of the plant. Reports compiled by [31] and [32] indicated the potential financial and environmental benefits of capturing and reusing waste

heat generated by food and drinks processing plants, particularly in the form 

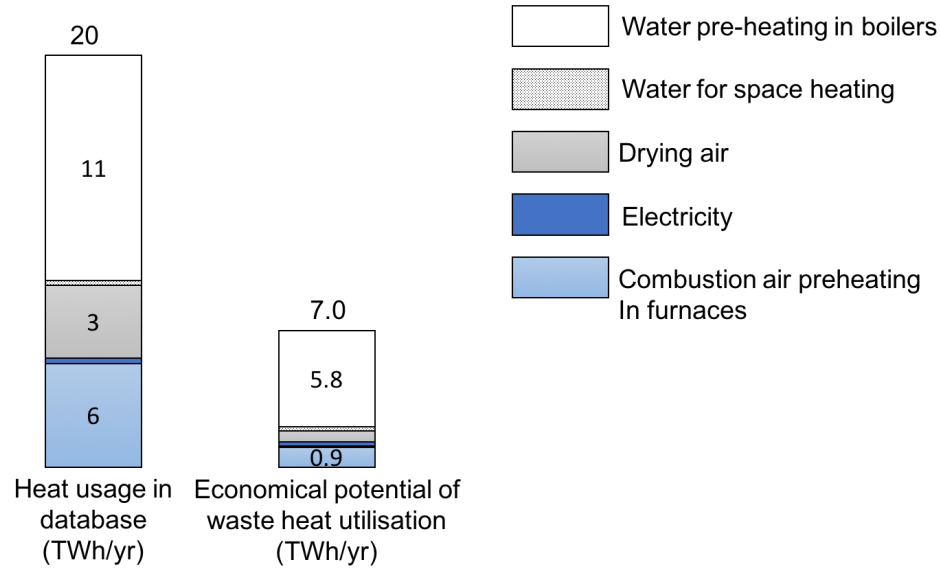

Figure 1: The breakdown of heat usage in the database compiled by [31, and the potential financial gain from the utilisation of waste heat.

of hot water. A summary is depicted in Figure 1, based on the information presented in [31.

Figure 2 illustrates a typical form of WHRS found within this sector. It is installed within a refrigeration system and coupled to a HWR. The WHRS, essentially a type of heat exchanger called a desuperheater, enables the exchange of heat from the hot refrigerant (the waste heat source) to the water (the heat sink), which is heated and stored in the HWR. The collected hot water can be used in a number of processes. A common use considered in the case study, is for cleaning, particularly within the plant's integrated internal cleaning process, known as clean-in-process or washdown.

\subsection{System parameters}

To ensure sufficient heat is captured by the WHRS, the difference between the temperature of the water and that of the refrigerant entering the WHRS should be large. Ideally, water from a mains supply will normally provide that large temperature difference, and would be sufficient to ensure significant waste heat is captured. This is ideal if the demand for hot water is in sync with when the waste heat is generated. However, like most renewable technologies, waste 


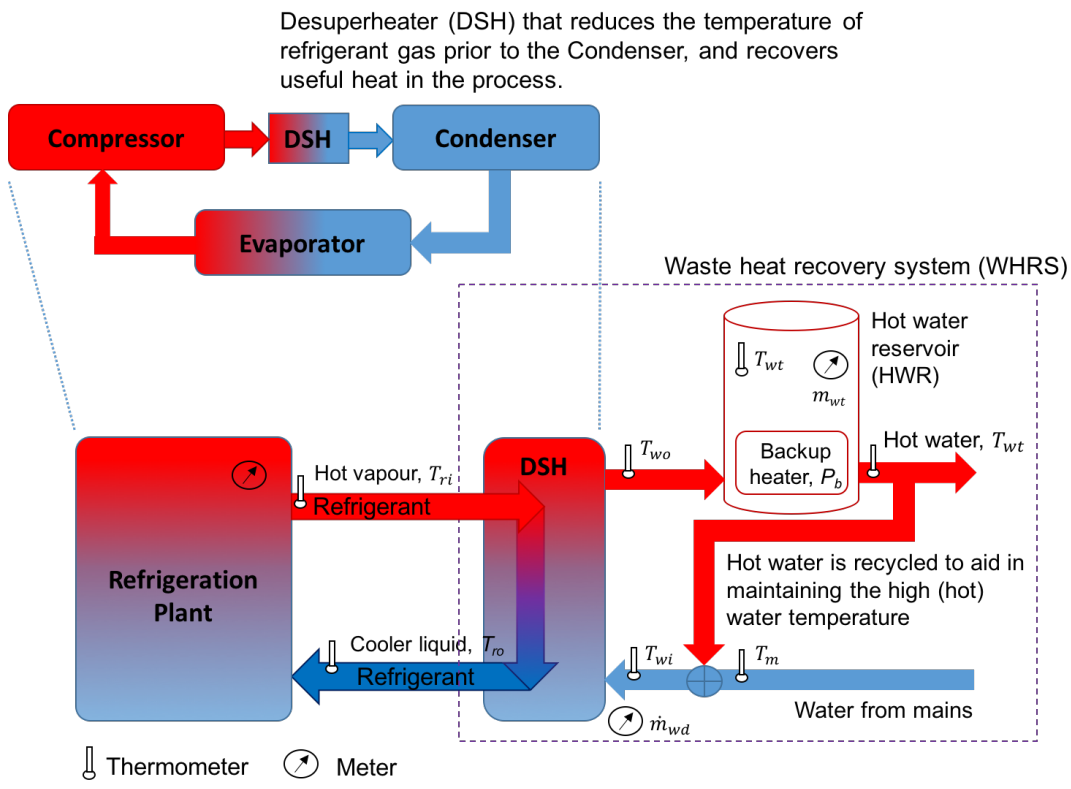

Figure 2: A waste heat recovery system comprising a desuperheater (DSH) and a hot water reservoir. Waste heat is provided by the refrigeration plant.

heat is often generated when the demand is low. For example, clean-in-process tends to occur at the beginning of the production process when there is relatively little need for refrigeration. Because of this, it is necessary to optimise the system in order to meet its anticipated needs. However, the optimisation of the WHRS comes with conflicting objectives affecting its configuration, especially regarding the design parameters of the HWR.

One key set of parameters concern the mass of water in the HWR: the minimum operating level $m_{w t_{\text {min }}}$ and the maximum operating level $m_{w t_{\max }}$. If the water volume is too low when demand takes place, then there may be a need to top up the volume in order to meet demand. This will require mains water to be heated by a back-up heater to bring the water temperature $\left(T_{w t}\right)$ up to the required operating temperature $\left(T_{h w}\right)$, bringing with it an associated energy cost. Hence, the $m_{w t_{\min }}$ should be sufficient to ensure that when the water is replenished from the mains water supply (to bring the volume up to $m_{w t_{\text {max }}}$ ), 
the water temperature is constantly maintained close to $T_{h w}$ using heat capture, with minimal injection of heat from the back-up heater. However, if $m_{w t_{\text {min }}}$ is high and the operating temperature is kept close to $T_{h w}$, then there will be less opportunity to capture waste heat by heating mains water. This is especially the case if the reservoir is relatively small, with a low $m_{w t_{\max }}$.

There will always be a loss of energy to the environment, in this case due to the temperature difference between the contents of the HWR and its surrounding region, which are not in equilibrium. With no heat source available, there is a continual drop in the HWR's water temperature. To ensure that no external heat is required when hot water is in demand, there is a need to capture an excess of heat in preparation for idle periods, which can be done by keeping the hot water temperature in the HWR $\left(T_{w t}\right)$ at a higher temperature, $T_{m x}$ (1). This ensures that $T_{h w}$ is met, despite the loss of heat to the environment $\left(T_{\text {loss }}\right)$. However, if $T_{m x}$ is too high, unanticipated demand requires cold water to be injected in order to reach $T_{h w}$.

$$
\Delta T_{\max }=T_{m x}-T_{h w}-T_{\text {loss }}
$$

Following from this, the parameters of the WHRS to be optimised are [30]:

1. $m_{w t_{\max }}$ : the maximum mass of water in the HWR, i.e. the capacity of the HWR,

2. $m_{w t_{m i n}}$ : the minimum mass of water that must be met when the hot water is demanded, also known as the depth of discharge (DoD),

3. $T_{m x}$ : the maximum temperature level of the HWR,

4. $P_{b_{\max }}$ : the maximum power of the back-up heater,

5. $\dot{m}_{w d_{\max }}$ : the maximum mass flow rate of the water entering the desuperheater (DSH).

\subsection{System objectives}

The following objectives were considered for optimising the WHRS [30]: 
1. to minimise the need for back-up energy when the heat captured by the WHRS is insufficient to meet demand,

2. to maximise the overall savings when using the WHRS, i.e. the difference in the external energy usage with and without the WHRS installation,

3. to minimise the temperature difference when the demanded temperature was not met,

4. to minimise the temperature difference when the HWR water temperature exceeds the demand,

5. to minimise the exceeding mass of water in the HWR from its maximum limit of $m_{w t_{\max }}$, when the water is replenished from the mains,

6. to minimise the waste heat not captured.

- Objectives 1, 3, 4 and 5 are motivated by the desire to reduce the overall cost of energy and water usage. Objectives 2, 4 and 6 reduce overall energy wastage, which in turn reduces $\mathrm{CO}_{2}$ emissions. Maximising objective 2 and minimising objective 6 can bring benefits from government incentive schemes that promote the reduction of carbon gas emission, for instance the UK CRC Energy Efficiency Scheme [34] and the EU Emissions Trading System (EU ETS) [35. These objectives may also gain benefits from other government incentives, such as the UK Ofgem Non-Domestic Renewable Heat Incentive (RHI) [36].

These objectives promote mutually conflicting design choices. Objective 1 , for instance, can be minimised by using a small reservoir; objectives 2 and 6 , by comparison, will potentially be optimised when a large reservoir is used. Objectives 5 and 6 benefit from a small $\dot{m}_{w d_{\max }}$ and $P_{b_{\max }}$; objective 3 benefits with small $\dot{m}_{w d_{\max }}$ and large $P_{b_{\max }}$; and objective 4 benefits with large $\dot{m}_{w d_{\max }}$ but small $P_{b_{\max }}$ 30].

\subsection{Simulation and optimisation}

In order to evaluate the impact of different design choices, a simulation model was constructed using Simulink ${ }^{\circledR}$. The model uses thermodynamics equations to simulate the temperature changes in the HWR given the discharge temperature 
of the refrigerant [37, the demand of the hot water, and the energy lost to the environment. A synthetic data set was created to model the demands for refrigeration (the waste heat source) and hot water (the waste heat sink) within this system, based upon patterns observed within two different dairy processing sites in Scotland.

Each time a design is evaluated, the simulation is run for a period of two working weeks, each week modelled upon one processing site, with the two patterns of demand concatenated to measure robustness (see Fig. 3), i.e. to ensure that the solutions obtained from optimisation are able to cope with varying demand. During the first week, waste heat is at its maximum capacity when there is no demand, and with similar time intervals (the first 5 work days or between $0 \mathrm{~s}$ to $4.32 \times 10^{5} \mathrm{~s}$ in Figure 3). To add variability, in the second half of the simulation (the next 5 work days or from $6.048 \times 10^{5} \mathrm{~s}$ onwards), the frequency of the refrigerant discharge was doubled with its intervals reduced by half of that of the hot water demand. The hot water flow rate is $2 \times 10^{3} \mathrm{~kg} / \mathrm{h}$ or $0.5556 \mathrm{~kg} / \mathrm{s}$, when demanded, indicated by the hot water demand signal in Figure 3 .

The simulations were performed with the values of $P_{b}$ depending on the temperature difference between the water $\left(T_{w i}\right)$ and the refrigerant $\left(T_{r i}\right)$ entering the WHRS. The value of $P_{b}$ is given by (2).

$$
\begin{gathered}
P_{b}=r_{p} \times P_{b_{\max }} \\
r_{p}= \begin{cases}1 & \text { if } r_{t_{p}}>0.75 \\
0.75 & \text { else if } 0.5<r_{t_{p}} \leq 0.75 \\
0.5 & \text { else if } 0.25<r_{t_{p}} \leq 0.5 \\
0.25 & \text { else if } 0<r_{t_{p}} \leq 0.25 \\
0 & \text { otherwise } \\
r_{t_{p}}=\frac{\left(T_{r i}-T_{w i}\right)}{T_{w i} \times 0.25}\end{cases}
\end{gathered}
$$



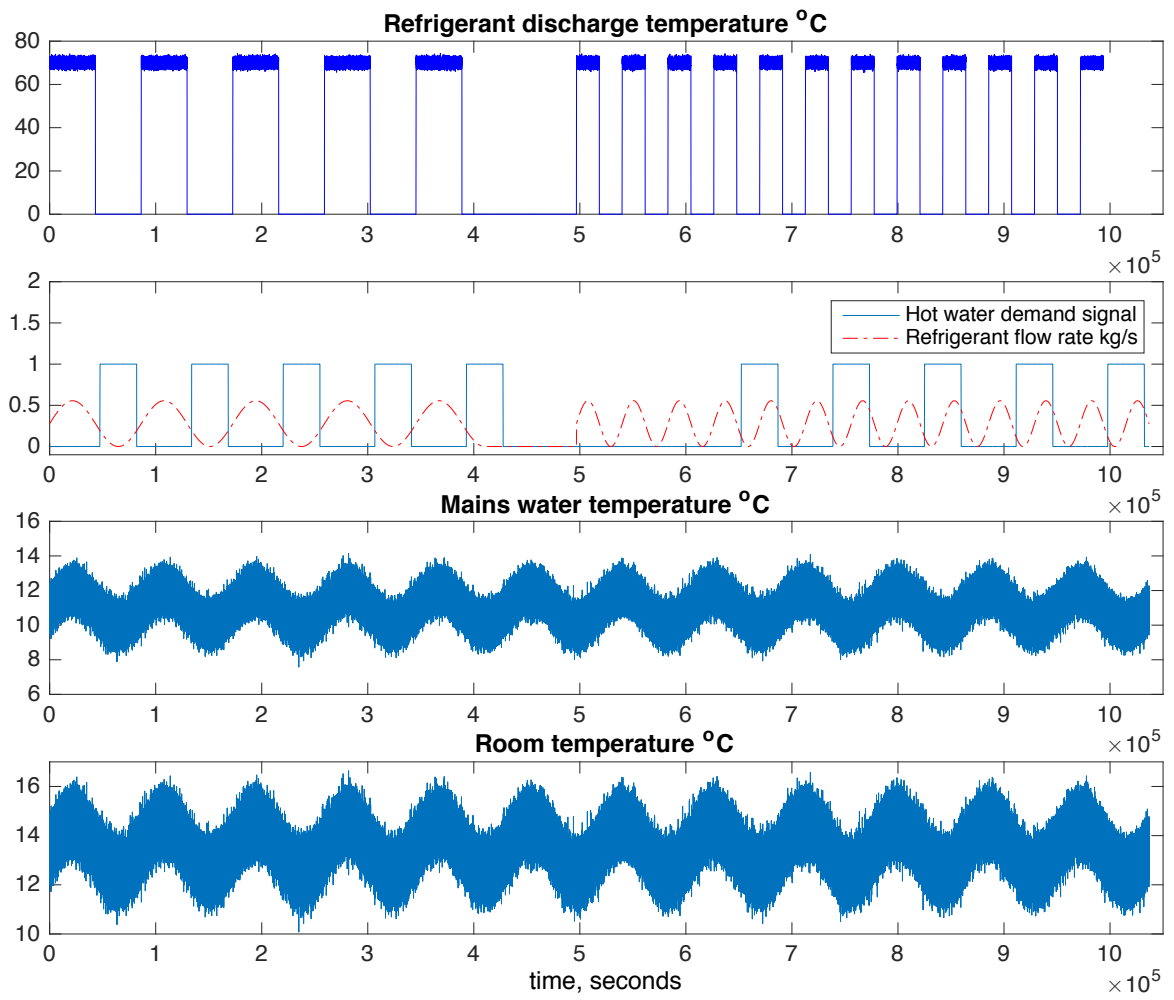

Figure 3: The properties of the refrigerant, hot water demand and the environmental conditions affecting the WHRS. The temperatures are simulated with added noise.

$\dot{m}_{w d}$ is dependent of the temperature difference between the temperature of the water in the HWR $\left(T_{w t}\right)$ and the desired temperature in the HWR $\left(T_{h w}\right) \cdot \dot{m}_{w d}$ is determined by $(5)$.

$$
\dot{m}_{w d}=r_{w d} \times \dot{m}_{w d_{\max }}
$$




$$
r_{w d}= \begin{cases}1 & \text { if } r_{t_{w}}>0.75 \\ 0.75 & \text { else if } 0.5<r_{t_{w}} \leq 0.75 \\ 0.5 & \text { else if } 0.25<r_{t_{w}} \leq 0.5 \\ 0.25 & \text { else if } 0<r_{t_{w}} \leq 0.25 \\ 0 & \text { otherwise } \\ r_{t_{w}}=\frac{\left(T_{h w}-T_{w t}\right)}{T_{h w} \times 0.5}\end{cases}
$$

Optimisation was carried out twice, first with a demand temperature $T_{h w}=$ $60^{\circ} \mathrm{C}$, a typical hot water temperature required for a food processing plant's clean-in-process, and the second time with $T_{h w}=40^{\circ} \mathrm{C}$, a water temperature that is used in newer clean-in-process solutions. NSGA-II was implemented in Matlab ${ }^{\circledR}$, using the typical NSGA-II variation operators settings taken from [27]: mutation probability $=1 /$ number of evolved parameters, distribution index for crossover $=20$, distribution index for mutation $=100$, population size $=200$, generations $=100$.

Table 1 lists the limits for the evolved parameters. The limits were added to bound the search space and to speed up the convergence of the algorithm. $m_{w t}$ and $T_{w t}$ are initialised with the evolved $m_{w t_{m a x}}$ at $T_{h w}$. Given that the simulation of the WHRS and HWR operations are time consuming due to its computational complexity, only one run of NSGA-II was performed and analysed for each of the two temperature demand scenarios.

\section{Results and Discussion}

A challenge of multiobjective optimisation is to find appropriate ways of visualising and understanding the resulting Pareto optimal sets. When the number of objectives is large $n>3$, as in this case study, it is not possible to directly plot the distribution of the optimised solutions based on their objectives' values, i.e. to plot the solutions within the objective space. This is the most common method of visualisation. To address this, a number of approaches 
Table 1: Limits for the evolved parameters.

\begin{tabular}{l|c|c}
\hline Parameter & $\min$ & $\max$ \\
\hline Maximum mass of water, $m_{w t_{\text {max }}}$ & $1.0 \times 10^{3} \mathrm{~kg}$ & $50.0 \times 10^{3} \mathrm{~kg}$ \\
Minimum mass of water, $m_{w t_{\text {min }}}(\%$ age of & $10 \%$ & $100 \%$ \\
$\left.m_{w t_{\text {max }}}\right)$ & & \\
Maximum difference in temperature, & $0^{\circ} \mathrm{C}$ & $98^{\circ} \mathrm{C}-T_{h w}$ \\
$\Delta T_{\text {max }}\left({ }^{\circ} \mathrm{C}\right)$ & & \\
Maximum power, $P_{b_{\text {max }}}(\mathrm{kW})$ & $6000 \mathrm{~kW}$ & $60000 \mathrm{~kW}$ \\
Maximum water flow rate, $\dot{m}_{w d_{\max }}(\mathrm{kg} / \mathrm{s})$ & $0.5 \mathrm{~kg} / \mathrm{s}$ & $1.0 \mathrm{~kg} / \mathrm{s}$ \\
\hline
\end{tabular}

are analysed. Three of these were considered: the use of summary metrics (hypervolumes), two dimensional cut-throughs (decision maps) and graphical representations (parallel coordinates).

\subsection{Hypervolumes}

Hypervolume is a measure of the compactness of a distribution of points within multidimensional space. When applied to objective space, it is commonly used as a summary metric for the optimum path and points between the constraints of the Pareto set found by an MOOA [38. Depending upon whether the problem is versed as a minimisation or maximisation problem, the aim is to either minimise or maximise the hypervolume between the Pareto set and the origin. Hypervolumes may also be used to describe convergence in parameter space, in which case convergence is indicated by a flattening-off of the hypervolume bounded by the solutions in the current Pareto set.

Figure 4 shows how the objective hypervolumes change over the course of the MOEA runs for the two temperature demand scenarios, when all objectives are normalised to be the maximisation problems. Figure 5 shows the corresponding hypervolumes in parameter space. Note that in both cases the variance between generations is due to stochasticity in the simulation. Figure 4 indicates that both runs converge to a similar hypervolume, suggesting that there 


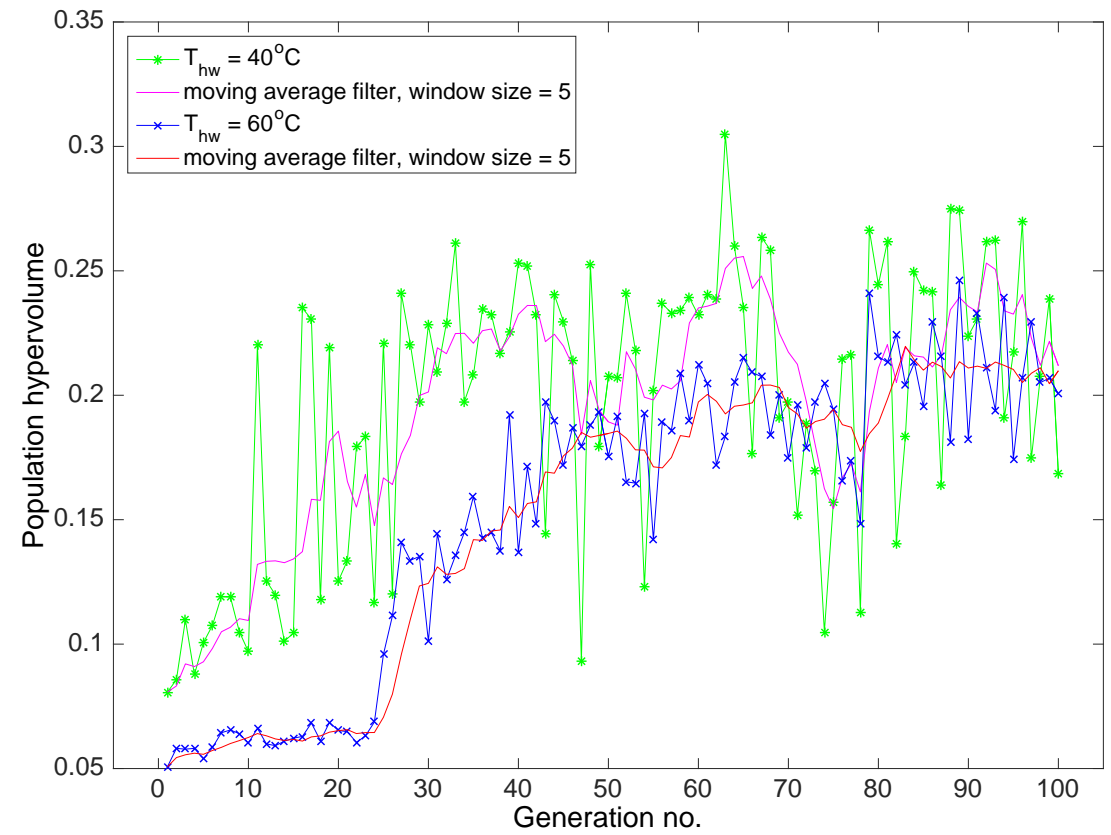

Figure 4: Objective hypervolumes of the Pareto set at each generation during the MOEA runs.

is no significant difference in the difficulty of the two problems, i.e. a change in demand temperature does not affect the difficulty of optimisation. Initial progress is faster for the $60^{\circ} \mathrm{C}$ scenario, but this may be due to the random sampling of the initial population by the MOEA. In general, it is not possible to tell whether the Pareto set found by the algorithm is equivalent to the true Pareto optimal set. Nevertheless, Figure 5 gives some more insight into the search behaviour. In particular, it can be seen that the algorithm explores an ever decreasing region of parameter space, and this region does not significantly shrink after about generation 80 . This suggests that it has identified the regions within which optimal solutions are found, and further optimisation then focuses on homing in on optimal values. 


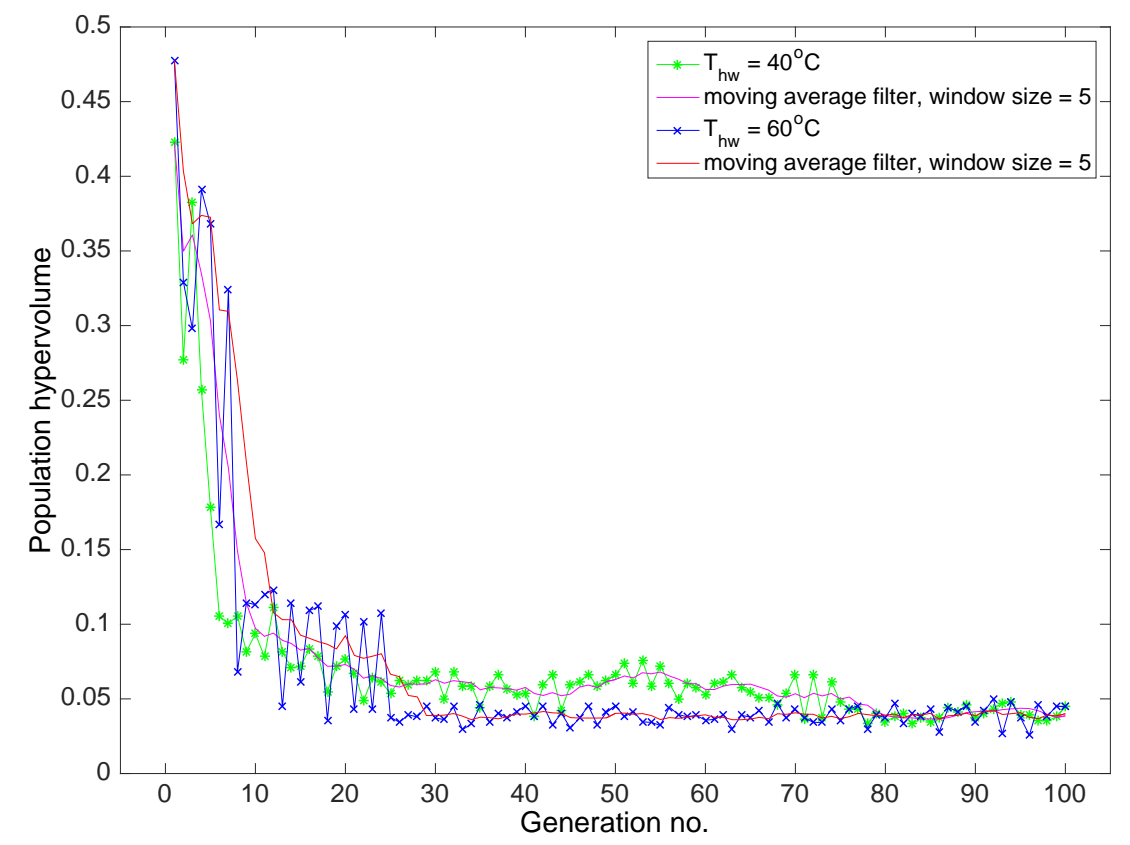

Figure 5: Parameter hypervolumes of the Pareto set at each generation during the MOEA runs.

\subsection{Decision Maps}

Hypervolumes can offer insight into the convergence to optimum solutions, and properties of the solution landscape, but they do not provide any information about the individual solutions. A more useful approach in this regard is to plot orthogonal two-dimensional projections of the multidimensional search space, showing either the trade-offs between objectives or the optimal regions of the parameter space. This is the most common method used for visualisation, as used in many MOOA studies [14, [11, [10], 15], 16].

These projections are also known as decision maps. For an $d$-dimensional space, there are $\left(\begin{array}{l}d \\ 2\end{array}\right)$ decision maps, and hence the number of maps grows rapidly. For the case study considered, which focuses on decision maps that depict the parameter space: with 5 parameters, there are 10 decision maps. A sample of these are shown in Figures 677 for the two different demand temperature sce- 

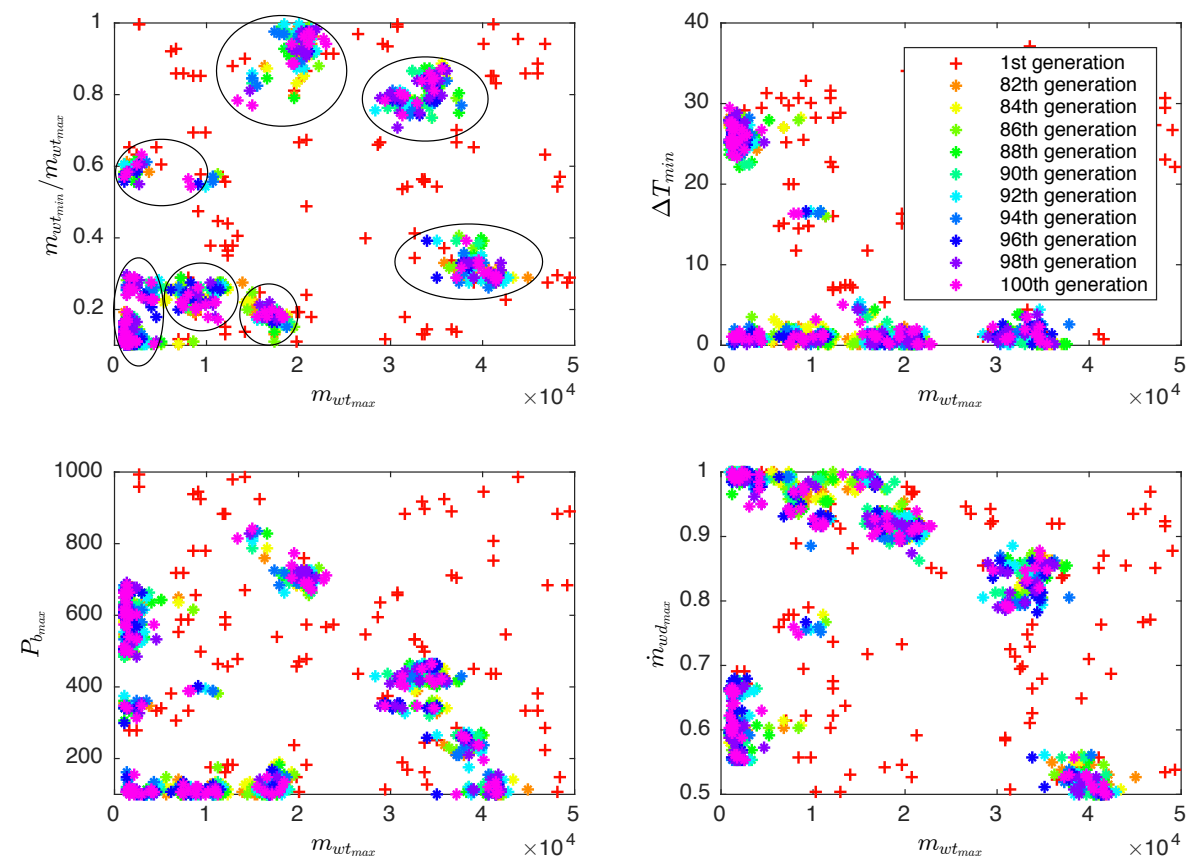

Figure 6: Two-dimensional projections of the evolved parameters of the solutions in the Pareto set for $T_{h w}=60^{\circ} \mathrm{C}$.

narios. In addition to depicting the Pareto set from the final 100th generation, the Pareto sets for the first and intermediate generations were also provided, showing how the MOEA has honed in on regions containing the optimal solutions.

It is notable that the decision maps for the two temperature demand scenarios are quite different. This suggests that the two optimisation spaces are topologically different, despite having similar levels of difficulty — something that was not evident from the hypervolume plots. For example, the decision map for the $T_{h w}=60^{\circ} \mathrm{C}$ case shown in Figure 6 indicates that the optimal solutions obtained favour solutions with smaller values (lower left hand side of the figures), in comparison to that of the $T_{h w}=40^{\circ} \mathrm{C}$ case. This, in turn, suggests a non-linear relationship between target temperature and parameters. Furthermore, by looking just at the parameters space alone, the correlation between the 

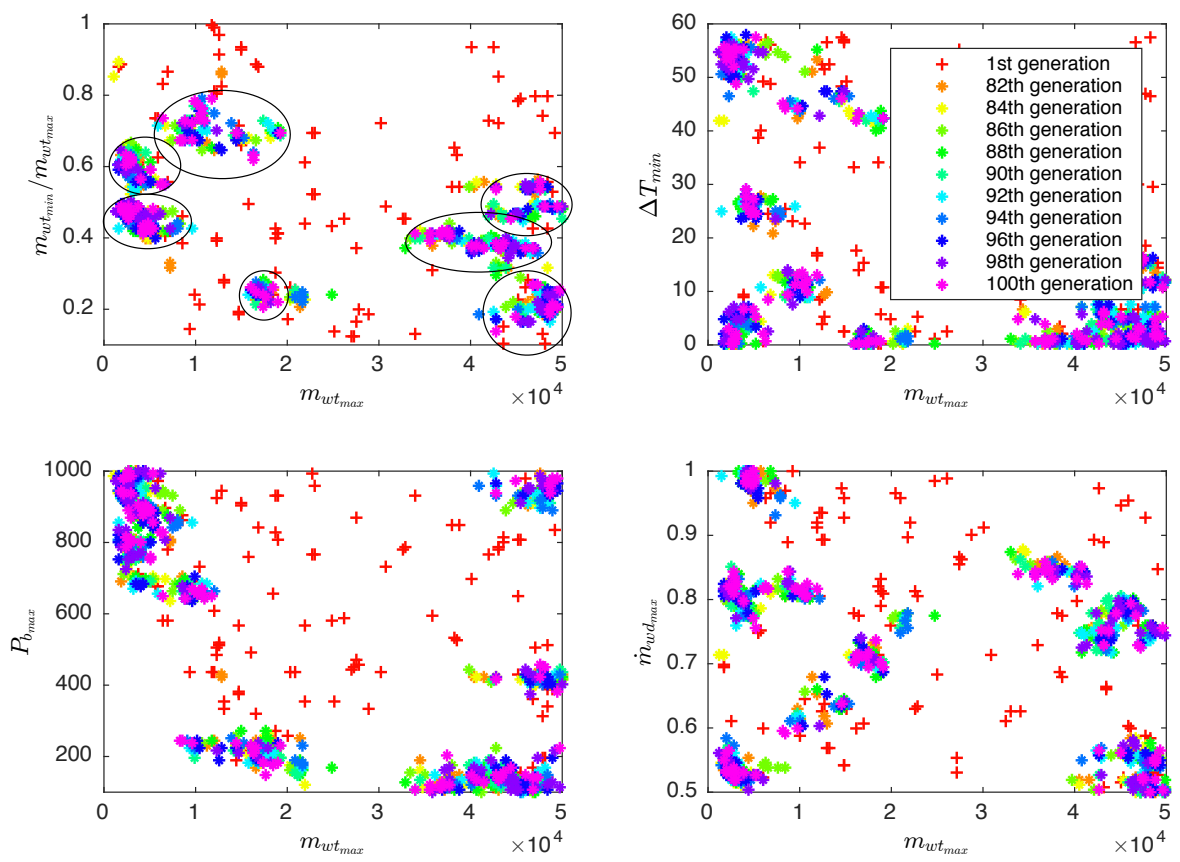

Figure 7: Two-dimensional projections of the evolved parameters of the solutions in the Pareto set for $T_{h w}=40^{\circ} \mathrm{C}$.

evolved parameters and their objective values are not shown. By looking at Figure 6 alone, one may assume that the optimal solutions will have small HWR tank size, for $T_{h w}=60^{\circ} \mathrm{C}$. Without correlating solutions to their objectives values, this may or may not be true.

It is also notable that the decision maps show a clustering of solutions within the search space (approximately indicated by circles). This suggests that there are a relatively small number of regions in which optimal solutions are found, and these represent a manageable number of installation types that exhibit different trade-offs between the objectives. These trade-offs are best evidenced when the solutions are visualised both in the parameter space and the objective space together. 


\subsection{Parallel Coordinates}

A weakness of decision maps is that they do not make it easy to elicit relationships between more than two parameters or objectives at once. As an alternative, parallel coordinate plots [24] were considered. Parallel coordinate plots are a visualisation method that transforms multidimensional patterns into a two-dimensional form by plotting the values from each dimension on parallel y-axes, as briefly introduced in Section 1. Parallel coordinate plots are similar to other two-dimensional transformation methods, such as Andrews curves and radar plots, that support data analysis by building on the human ability to distinguish two-dimensional forms.

The parallel coordinates plot consists of $d$-number of parallel lines, each line corresponding to one of the axis of the $d$-dimensional data. A point in a $d$ dimensional space will be visualised on the parallel coordinates plot as a single horizontal line that connects a point on each of the parallel lines. A point on the parallel line is a coordinate of the solution at a specific axis. Given that each parallel line in itself is an individual axis, the parallel lines may not have the same scale as one another. In simplest term, the parallel coordinates plot is similar to the concatenation of $d$-number of two-dimensional plots, with the parallel coordinates plot consisting of $d$-number of $\mathrm{y}$-axes, and the $\mathrm{x}$-axis is the index for each of the individual plots.

Following the observed clustering of solutions in the decision maps, the $k$ means clustering algorithm was used to group the solutions into $k=7$ clusters. Figure 8 shows the parallel coordinate plots for the Pareto-optimal solutions clustered in the objective space, and in Figure 9 the solutions are clustered in the parameter space. Because of this, solutions in each cluster will have similar attributes, either in terms of objective values or parameter values. Using separate parallel coordinate plots for each cluster makes it easier to visualise and analyse the space of designs.

To avoid clutter, the y-axes are not shown for each dimension; rather, all values are normalised and scaled to the interval $[0,1]$, allowing the use of a shared y-axis. All clusters also share the same limits used in the normalisation, 
and the limits for each objective and parameter are detailed in Tables 1 and 2 . The correlation between a solution and its objectives values in a specific cluster is distinguishable by the common colour used in both plots.

In most cases, the same patterns are seen regardless of whether clustering is performed in objective or parameter space, and in general these have well defined two-dimensional shapes when visualised with parallel coordinates. This suggests that there is a distinct and manageable set of alternative designs within the Pareto set. However, there are some notable differences between the clusterings in the two spaces. For instance, in the $T_{h w}=40^{\circ} \mathrm{C}$ case, clusters 6 and 7 in objective space (Figure 8a) are combined into cluster 6 in parameter space (Figure 9a). Cluster 3 in the objective space is split into clusters 3 and 7 in the parameter space. For $T_{h w}=60^{\circ} \mathrm{C}$ case, cluster 6 in objective space (Figure $8 \mathrm{~b}$ is split into clusters 6 and 7 in parameter space (Figure $9 \mathrm{~b}$ ). This shows the value of generating clusterings and visualisations in both the objective space and the parameter space. The figures also show how parameter variability within each group affects the objective values.

A visual inspection of Figure 9 indicates that only one two-dimensional pattern occurs in the Pareto sets for both temperature demand scenarios: this occurs as cluster 5 in Figure $9 \mathrm{a}$ and cluster 2 in Figure $9 \mathrm{~b}$. This corresponds to installations with small $m_{w t_{\max }}$, high $m_{w t_{\min }}$, high $T_{m x}$, low $P_{b_{\max }}$ and high $\dot{m}_{w d_{\max }}$. This is hence a desirable solution if there is a likelihood of the demand temperature changing in the future-for instance, if the plant's clean-in-process (CIP) uses a CIP solution able to operate at lower temperature. However, it should be noted that although it ranks highly in terms of the maximum capture of waste heat (objective 5), this flexibility comes with a trade-off, in particular for the $60^{\circ} \mathrm{C}$ temperature demand scenario: (objective 1) high cost of external energy usage and (objective 2) low savings achieved.

\subsubsection{Clustering in the objective space}

In general, the results show that in order to save on running costs, a large hot water tank is desirable. However, larger tanks imply higher installation 

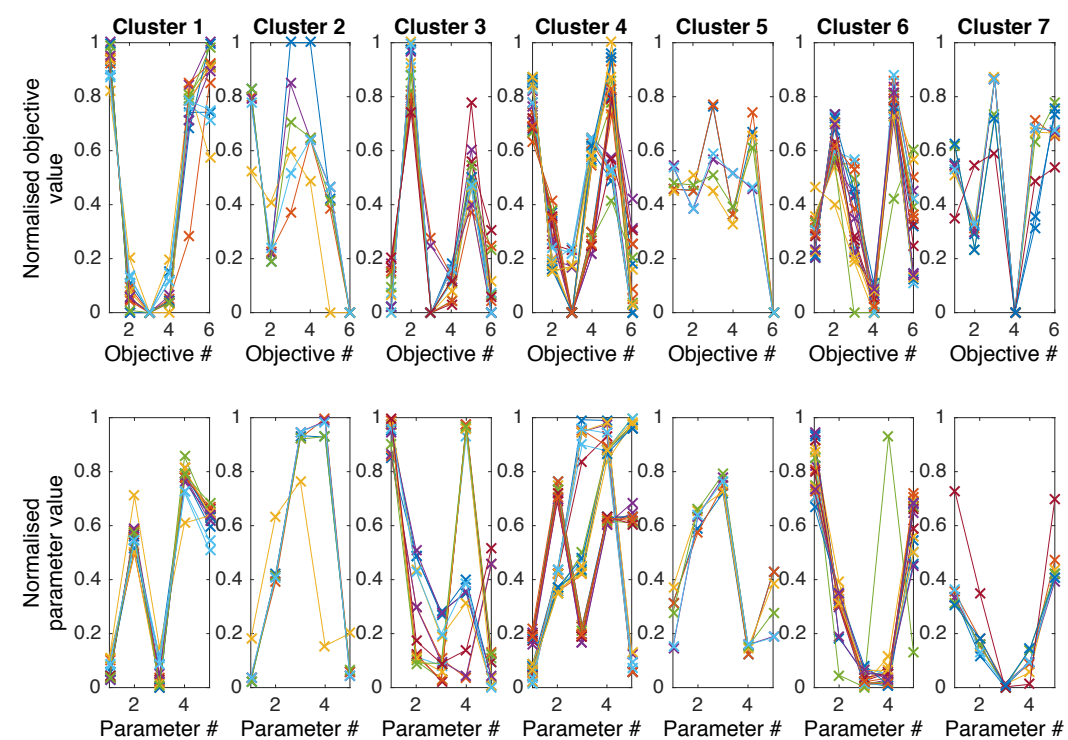

(a) $T_{h w}=40^{\circ} \mathrm{C}$
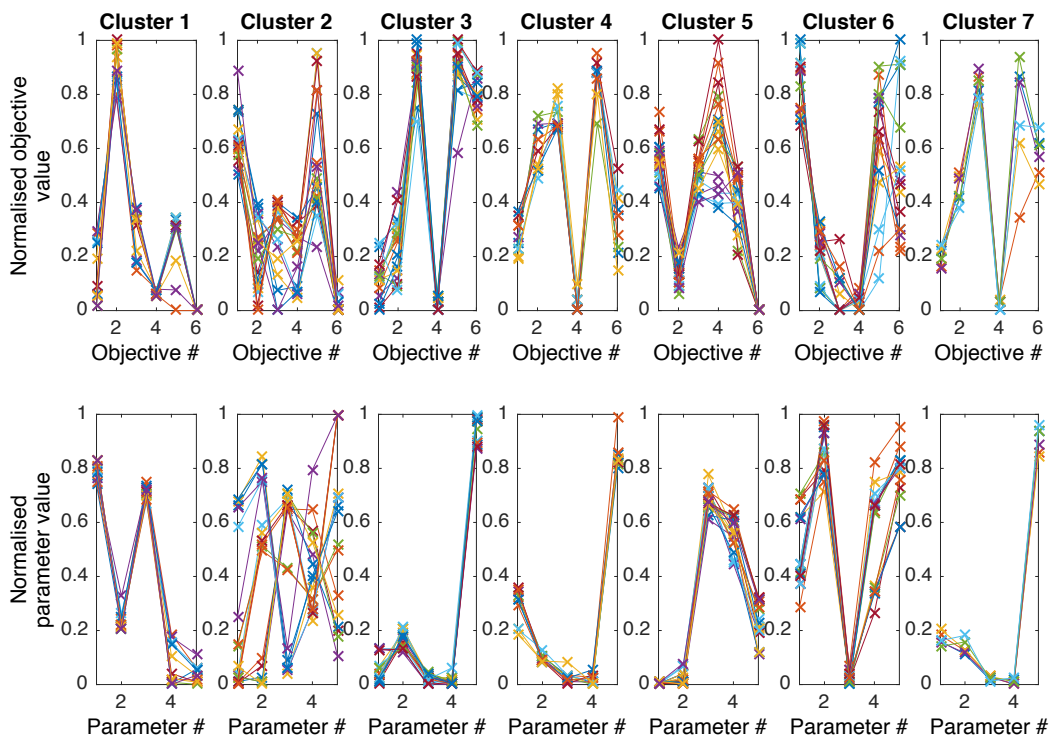

(b) $T_{h w}=60^{\circ} \mathrm{C}$

Figure 8: Parallel coordinate plots showing clusters of solutions in the Pareto set when clustering is carried out in objective space. Each solution in a cluster is indicated by a different coloured line. 

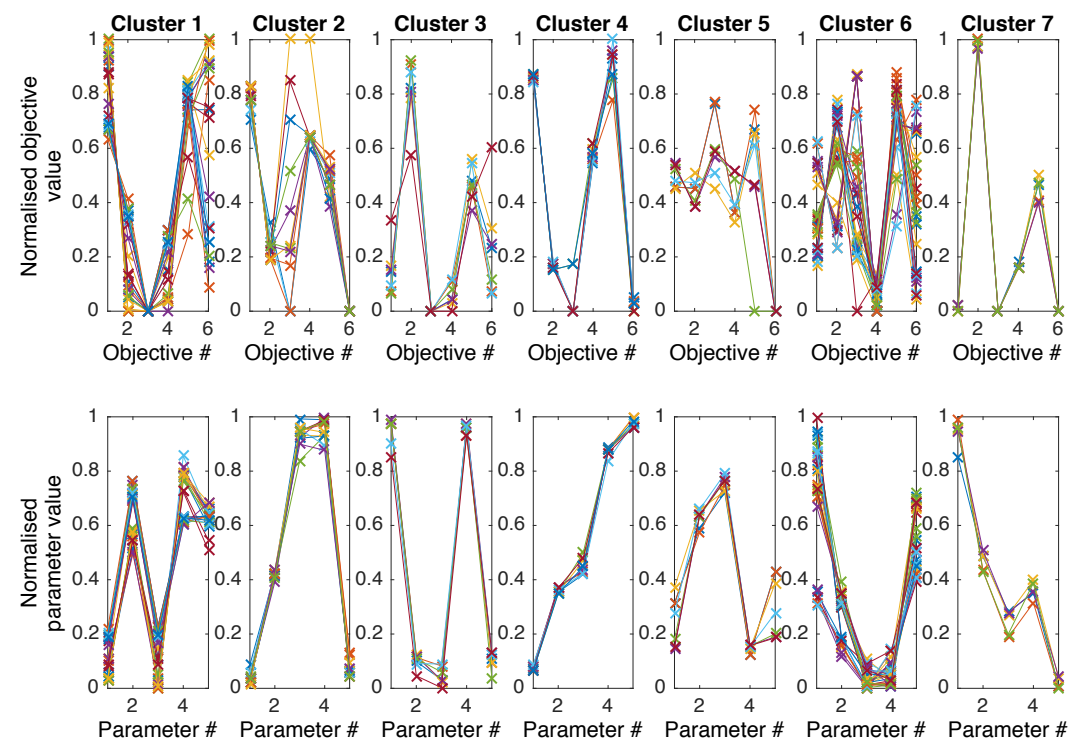

(a) $T_{h w}=40^{\circ} \mathrm{C}$
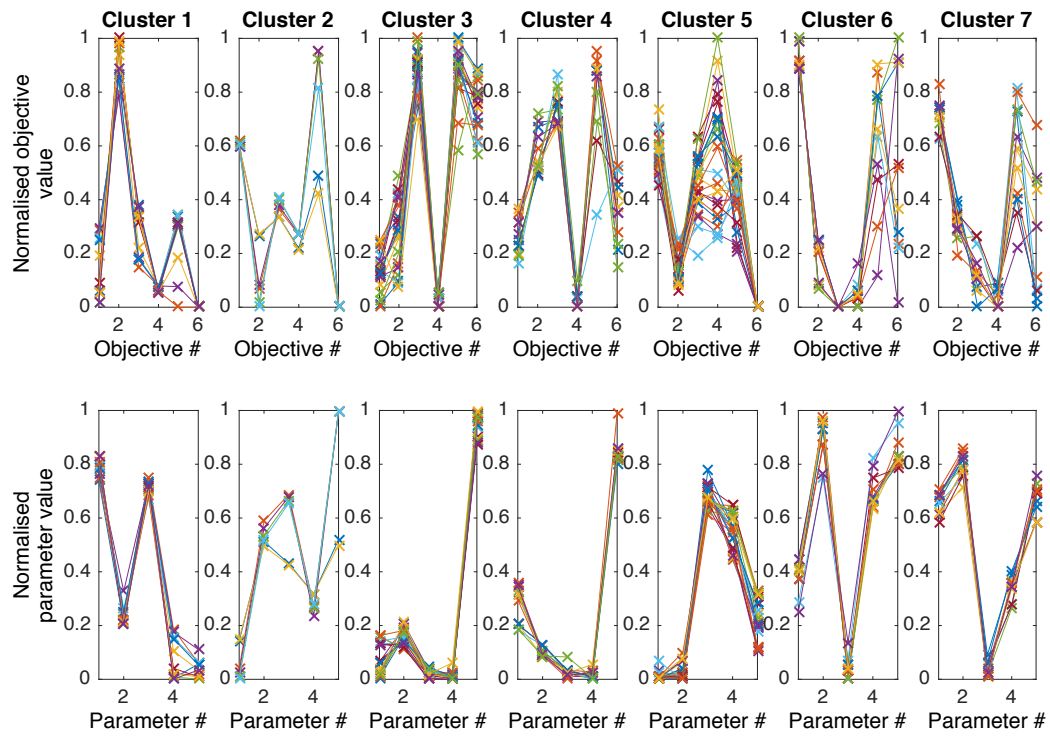

(b) $T_{h w}=60^{\circ} \mathrm{C}$

Figure 9: Parallel coordinate plots showing clusters of solutions in the Pareto set when clustering is carried out in parameter space. Each solution in a cluster is indicated by a different coloured line. 
Table 2: The max and min values used for normalisation of the objectives' values in Figures $8 \mathrm{a}-9 \mathrm{a}$

\begin{tabular}{|c|c|c|c|c|}
\hline \multirow[b]{2}{*}{ Objectives } & \multicolumn{2}{|c|}{$60^{\circ} \mathrm{C}$} & \multicolumn{2}{|c|}{$40^{\circ} \mathrm{C}$} \\
\hline & Max & Min & Max & Min \\
\hline $\begin{array}{l}\text { 1. To minimise } \\
\text { back-up energy }\end{array}$ & $10.356 \mathrm{MWh}$ & $6.5257 \mathrm{MWh}$ & $5.7793 \mathrm{MWh}$ & $2.5120 \mathrm{MWh}$ \\
\hline $\begin{array}{l}\text { 2. To maximise } \\
\text { the savings }\end{array}$ & $27.20 \%$ & $2.20 \%$ & $57.01 \%$ & $13.39 \%$ \\
\hline $\begin{array}{l}3 . \text { To min- } \\
\text { imise the tem- } \\
\text { perature differ- } \\
\text { ence with the } \\
\text { demand temper- } \\
\text { ature was not } \\
\text { met }\end{array}$ & $22.4781{ }^{\circ} \mathrm{C}$ & $0^{\circ} \mathrm{C}$ & $5.8854{ }^{\circ} \mathrm{C}$ & $0{ }^{\circ} \mathrm{C}$ \\
\hline $\begin{array}{l}4 . \quad \text { To min- } \\
\text { imise the tem- } \\
\text { perature differ- } \\
\text { ence with the } \\
\text { demand temper- } \\
\text { ature has been } \\
\text { exceeded }\end{array}$ & $31.8732{ }^{\circ} \mathrm{C}$ & $0^{\circ} \mathrm{C}$ & $44.2305^{\circ} \mathrm{C}$ & $0^{\circ} \mathrm{C}$ \\
\hline $\begin{array}{l}5 . \text { To minimise } \\
\text { the exceeding } \\
\text { mass of water in } \\
\text { the HWR }\end{array}$ & $58.4071 \mathrm{~kg}$ & $16.6283 \mathrm{~kg}$ & $56.7865 \mathrm{~kg}$ & $2.6323 \mathrm{~kg}$ \\
\hline $\begin{array}{l}\text { 6. To minimise } \\
\text { the waste heat } \\
\text { not captured }\end{array}$ & $38.98 \%$ & $0 \%$ & $41.66 \%$ & $0 \%$ \\
\hline
\end{tabular}


costs. Although not an objective considered in this study, as installation cost was not one of the driving factor for the process industries involved. Initial cost may be a significant concern for some installations. The research results still give an insight into the trade-offs involved. In particular, for the $T_{h w}=60^{\circ} \mathrm{C}$ temperature demand scenario, if a small tank is preferable with small running cost (external energy used), a solution is also available from the Pareto-optimal set (clusters 3 and 4 in Figure 9b. This however, is at the expense of the amount of waste heat recovered, and in turn, in the amount of savings achieved, if one was to also compare these clusters with that of cluster 1 in Figure $9 \mathrm{~b}$, consisting of solutions with larger tank sizes. This similar observation was made in [7, whereby, if payback period was a priority, the solution will come at the expense of its efficiency and effectiveness of the waste heat recovery. If a small tank is, however, required, solutions in cluster 4 provide a better investment in comparison to those in cluster 3 .

A similar comparison can also be shown for $T_{h w}=40^{\circ} \mathrm{C}$. By sacrificing on the cost of installation through the installation of a larger tank, more savings can be achieved in the long run. This can be shown by comparing the solutions in cluster 6 with those in cluster 7 in Figure 8 a. Figure 8 a also indicates that solutions in cluster 3 with large HWR tank size have higher savings achieved, low external energy used and maximum waste heat captured. If a small tank is preferable, solutions in cluster 5 provides better options.

\subsubsection{Clustering in the parameter space}

The clusters in parameter space are generally more distinct than those in objective space. This suggests that some of the parameters are sensitive, i.e. small changes to their values can lead to comparatively large changes in objective values. Interestingly, this distinctness remains when the parameter cluster members are determined by clusterings done in objective space rather than the parameter space. This highlights the existence of relatively small regions of good parameter choices, both reflecting the analysis of the decision maps and indicating the importance of carrying out optimisation to identify these small 
regions of optimal behaviour.

The proposed visualisation provides the ability to analyse the impact of changes to the parameters. Parameter sensitivity within the clusters provides additional information to the installer. The MOEA is tasked with optimising the physical system configuration of the WHRS; however, these parameters can be changed in real-time through software, provided that the values required do not exceed the maximum limits of the installed system. For example, if the installer was to fit the maximum tank size with the maximum rated power for the backup heater and the maximum pipe size which will allow for the maximum mass flow rate of water to flow to the desuperheater from an identified cluster, the WHRS software could then be configured to set the operational limits of the system based on the actual conditions within which the WHRS is operating.

If the WHRS were to be configured in software, information on how the change in parameters can affect their objectives is useful. The presented visualisation provides this information, particularly within clusters that display parameter instability. In this case, the instability of parameters was especially evident when the WHRS is operating at its limits. For instance, cluster 5 in Figures $8 \mathrm{~b}$ and $9 \mathrm{~b}$ shows a solution in which the tank size is small, there is a low minimum water level when in demand, the flow rate is low, and the backup heater power is high. It is evident from the corresponding view in objective space that, in this situation, a small range of parameter values lead to a wide range of objective values. This is especially the case for objective 4 , the likelihood of the temperature exceeding the target. In this case, the research concluded that the system has a limited capacity to adapt to changes in the pattern of demand. The capacity for a multiobjective analysis such as this to highlight these kind of situations is useful, since in practice installers are likely to want to avoid choices that lead to unstable behaviour. 


\section{Conclusions}

This paper shows how a multiobjective evolutionary algorithm (MOEA) in concert with multidimensional visualisation methods can be used to explore the design space of a waste heat recovery system (WHRS), which recovers waste heat to provide hot water stored in a hot water reservoir (HWR) at two different hot water temperature requirements. Unlike conventional optimisation approaches, most of which return a single solution to a given problem, multiobjective approaches explicitly identify solutions with different trade-offs, providing a broader view of possible design choices. This is particularly important when optimisation objectives are mutually exclusive, as in the industrial case study described in this paper.

The MOEA was first used to find the Pareto-optimal solutions. Clustering then identified the relatively small number of representative trade-off solutions that could be considered by potential installers. Parallel coordinate plots were used to visualise these designs, helping to better understand the trade-offs within the design space, and to identify parameter sensitivities that may be considered during the installation of a WHRS. In the case study presented, if one was to prioritise the minimisation of cost through the installation of a small HWR, this may impact on the effectiveness and efficiency of the WHRS in recovering waste heat, and in turn the saving achieved. This trade-off is made obvious when the Pareto-optimal solutions and their objectives were displayed using the proposed visualisation methods. The results from this case study also illustrate the broader benefits that could be achieved by applying multi-objective methods, in concert with with clear visualisations of the Pareto-optimal solutions, to industrial systems design, both in the energy sector and more broadly.

\section{Acknowledgment}

The authors would like to thank Waste Heat Recovery Limited, Mackie's of Scotland, Müller Milk and Ingredients, and Neil Urquhart for their input. This 
work was supported by Innovate UK (project no. 101995) and the Engineering and Physical Sciences Research Council (EP/M507180/1).

[1] T. G. Walmsley, M. R. Walmsley, M. J. Atkins, J. R. Neale, Integration of industrial solar and gaseous waste heat into heat recovery loops using constant and variable temperature storage, Energy 75 (2014) 53 - 67 . doi:http://dx.doi.org/10.1016/j.energy.2014.01.103

URL http://www.sciencedirect.com/science/article/pii/ S036054421400125X

[2] S. S. Cipolla, M. Maglionico, Heat recovery from urban wastewater: Analysis of the variability of flow rate and temperature, Energy and Buildings 69 (2014) $122-130$.

[3] G. Zavala, A. J. Nebro, F. Luna, C. A. Coello Coello, Structural design using multi-objective metaheuristics. comparative study and application to a real-world problem, Structural and Multidisciplinary Optimization 53 (3) (2016) 545-566. doi:10.1007/s00158-015-1291-3. URL http://dx.doi.org/10.1007/s00158-015-1291-3

[4] A. Arias-Montano, C. A. C. Coello, E. Mezura-Montes, Multiobjective evolutionary algorithms in aeronautical and aerospace engineering, IEEE Transactions on Evolutionary Computation 16 (5) (2012) 662-694. doi: 10.1109/TEVC.2011.2169968

[5] A. L. Jaimes, C. Coello, Multi-objective evolutionary algorithms: A review of the state-of-the-art and some of their applications in chemical engineering, Multi-Objective Optimization: Techniques and Applications in Chemical Engineering (2009) 61-86.

[6] C. A. C. Coello, G. B. Lamont, Applications of multi-objective evolutionary algorithms, Vol. 1, World Scientific, 2004.

[7] C. Zhang, L. Zhou, P. Chhabra, S. S. Garud, K. Aditya, A. Romagnoli, G. Comodi, F. D. Magro, A. Meneghetti, M. Kraft, 
A novel methodology for the design of waste heat recovery network in eco-industrial park using techno-economic analysis and multi-objective optimization, Applied Energy 184 (2016) 88 - 102. doi:http://dx.doi.org/10.1016/j.apenergy.2016.10.016.

URL http://www.sciencedirect.com/science/article/pii/ S0306261916314404

[8] V. Tulus, D. Boer, L. F. Cabeza, L. Jiménez, G. GuillénGosálbez, Enhanced thermal energy supply via central solar heating plants with seasonal storage: A multi-objective optimization approach, Applied Energy 181 (2016) 549 - 561. doi:http: //dx.doi.org/10.1016/j.apenergy.2016.08.037

URL http://www.sciencedirect.com/science/article/pii/ S0306261916311242

[9] N. Nazari, P. Heidarnejad, S. Porkhial, Multi-objective optimization of a combined steam-organic rankine cycle based on exergy and exergo-economic analysis for waste heat recovery application, Energy Conversion and Management 127 (2016) 366 - 379. doi:http://dx.doi.org/10.1016/j.enconman.2016.09.022.

URL http://www.sciencedirect.com/science/article/pii/ S0196890416308007

[10] M. Khoroshiltseva, D. Slanzi, I. Poli, A pareto-based multiobjective optimization algorithm to design energy-efficient shading devices, Applied Energy 184 (2016) 1400 - 1410. doi:http: //dx.doi.org/10.1016/j.apenergy.2016.05.015 URL http://www.sciencedirect.com/science/article/pii/ S0306261916305979

[11] T. Falke, S. Krengel, A.-K. Meinerzhagen, A. Schnettler, Multiobjective optimization and simulation model for the design of distributed energy systems, Applied Energy 184 (2016) 1508 - 1516. 
doi:http://dx.doi.org/10.1016/j.apenergy.2016.03.044.

URL http://www.sciencedirect.com/science/article/pii/ S0306261916303646

[12] M. Braun, T. Dengiz, I. Mauser, H. Schmeck, Comparison of multiobjective evolutionary optimization in smart building scenarios, in: Applications of Evolutionary Computation: 19th European Conference, EvoApplications 2016, Porto, Portugal, March 30 - April 1, 2016, Proceedings, Springer International Publishing, 2016.

[13] S. Fazlollahi, G. Becker, A. Ashouri, F. Maréchal, Multi-objective, multiperiod optimization of district energy systems: $\{\mathrm{IV}\}$ - a case study, Energy 84 (2015) 365 - 381. doi:http://dx.doi.org/10.1016/j.energy. 2015. 03.003 .

URL http://www.sciencedirect.com/science/article/pii/ S0360544215002856

[14] M. S. Mahbub, M. Cozzini, P. A. Østergaard, F. Alberti, Combining multi-objective evolutionary algorithms and descriptive analytical modelling in energy scenario design, Applied Energy 164 (2016) 140 - 151. doi:http://dx.doi.org/10.1016/j.apenergy.2015.11.042.

URL http://www.sciencedirect.com/science/article/pii/ S0306261915014920

[15] D. H. Muhsen, A. B. Ghazali, T. Khatib, I. A. Abed, E. M. Natsheh, Sizing of a standalone photovoltaic water pumping system using a multi-objective evolutionary algorithm, Energy 109 (2016) 961 - 973. doi:http://dx.doi.org/10.1016/j.energy.2016.05.070.

URL http://www.sciencedirect.com/science/article/pii/ S0360544216306879

[16] S. Lotfan, R. A. Ghiasi, M. Fallah, M. Sadeghi, Ann-based modeling and reducing dual-fuel engine's challenging emissions by multi-objective evolutionary algorithm nsga-ii, Applied Energy 175 (2016) 91 - 99. 
doi:http://dx.doi.org/10.1016/j.apenergy.2016.04.099.

URL http://www.sciencedirect.com/science/article/pii/ S030626191630561X

[17] J. Wang, Z. Yan, M. Wang, M. Li, Y. Dai, Multi-objective optimization of an organic rankine cycle (orc) for low grade waste heat recovery using evolutionary algorithm, Energy Conversion and Management 71 (2013) 146 - 158. doi:http://dx.doi.org/10.1016/j.enconman.2013.03.028

URL http://www.sciencedirect.com/science/article/pii/ S0196890413001714

[18] M. Escobar, J. O. Trierweiler, Optimal heat exchanger network synthesis: A case study comparison, Applied Thermal Engineering 51 (1-2) (2013) 801 - 826. doi:http://dx.doi.org/10.1016/j.applthermaleng. 2012 . 10.022

URL http://www.sciencedirect.com/science/article/pii/ S1359431112006783

[19] A. Isafiade, D. Fraser, Interval based $\{$ MINLP $\}$ superstructure synthesis of heat exchanger networks for multi-period operations, Chemical Engineering Research and Design 88 (10) (2010) 1329 - 1341. doi:http://dx.doi.org/10.1016/j.cherd.2010.02.019 URL http://www.sciencedirect.com/science/article/pii/ S0263876210000870

[20] J. Stamp, T. Majozi, Optimum heat storage design for heat integrated multipurpose batch plants, Energy 36 (8) (2011) 5119 - 5131.

[21] K. feng Huang, I. Karimi, Efficient algorithm for simultaneous synthesis of heat exchanger networks, Chemical Engineering Science 105 (2014) 53 -68. doi:http://dx.doi.org/10.1016/j.ces.2013.10.040.

URL http://www.sciencedirect.com/science/article/pii/ S0009250913007276 
[22] A. Jiménez-Gutiérrez, J. Lona-Ramírez, J. M. Ponce-Ortega, M. El-Halwagi, An \{MINLP\} model for the simultaneous integration of energy, mass and properties in water networks, Computers \& Chemical Engineering 71 (2014) 52 - 66. doi:http: //dx.doi.org/10.1016/j.compchemeng.2014.07.008.

URL http://www.sciencedirect.com/science/article/pii/ S0098135414002129

[23] C.-L. Chen, Y.-J. Ciou, Design of indirect heat recovery systems with variable-temperature storage for batch plants, Industrial \& Engineering Chemistry Research 48 (9) (2009) 4375-4387. arXiv:http://dx.doi.org/ 10.1021/ie8013633, doi:10.1021/ie8013633.

URL http://dx.doi.org/10.1021/ie8013633

[24] A. Inselberg, Parallel Coordinates: Visual Multidimensional Geometry and Its Applications, Springer-Verlag New York, Inc., Secaucus, NJ, USA, 2009.

[25] A. Inselberg, Multidimensional detective, in: Information Visualization, 1997. Proceedings., IEEE Symposium on, 1997, pp. 100-107. doi:10. 1109/INFVIS.1997.636793.

[26] M. A. Lones, Metaheuristics in nature-inspired algorithms, in: Proceedings of the Companion Publication of the 2014 Annual Conference on Genetic and Evolutionary Computation, GECCO Comp '14, ACM, New York, NY, USA, 2014, pp. 1419-1422. doi:10.1145/2598394.2609841

URL http://doi.acm.org/10.1145/2598394.2609841

[27] K. Deb, A. Pratap, S. Agarwal, T. Meyarivan, A fast and elitist multiobjective genetic algorithm: Nsga-ii, Evolutionary Computation, IEEE Transactions on 6 (2) (2002) 182-197. doi:10.1109/4235.996017.

[28] A. Alarcon-Rodriguez, G. Ault, S. Galloway, Multi-objective planning of distributed energy resources: A review of the state-of-the-art, Renewable and Sustainable Energy Reviews 14 (5) (2010) 1353 - 1366. 
doi:http://dx.doi.org/10.1016/j.rser.2010.01.006

URL http://www.sciencedirect.com/science/article/pii/ S1364032110000146

[29] R. Evins, A review of computational optimisation methods applied to sustainable building design, Renewable and Sustainable Energy Reviews 22 (2013) 230 - 245. doi:http://dx.doi.org/10.1016/j.rser.2013. 02.004

URL http://www.sciencedirect.com/science/article/pii/ S1364032113000920

[30] M. Mokhtar, I. Hunt, S. Burns, D. Ross, Optimising a waste heat recovery system using multi-objective evolutionary algorithm, in: Proceedings of the 2016 on Genetic and Evolutionary Computation Conference Companion, GECCO '16 Companion, ACM, New York, NY, USA, 2016, pp. 913-920.

[31] The potential for recovering and using surplus heat from industry, Tech. rep., Element Energy Limited, Ecofys, Imperial College London, Dr. Paul Stevenson and Dr. Robert Hyde (2014).

URL https://www.gov.uk/government/publications/ the-potential-for-recovering-and-using-surplus-heat-from-industry

[32] Industrial energy efficiency accelerator: Guide to the dairy sector, Tech. rep., Carbon Trust (2011).

URL http://www . carbontrust. com/media/206472/ ctg033-dairy-industrial-energy-efficiency.pdf

[33] Carbon budgets and targets (2015).

URL https://www.theccc.org.uk/tackling-climate-change/ reducing-carbon-emissions/carbon-budgets-and-targets/

[34] Crc energy efficiency scheme: charging scheme and guidance (2016).

URL https://www.gov.uk/government/publications/

carbon-reduction-commitment-crc-energy-efficiency-scheme-april-2013-to-march-2014 
[35] The eu emissions trading system (eu ets).

URL http://ec.europa.eu/clima/policies/ets/index_en.htm

[36] Non-domestic renewable heat incentive (rhi) (2016).

URL https://www.ofgem.gov.uk/environmental-programmes/ non-domestic-rhi

[37] D. T. Reindl, T. B. Jekel, Heat recovery in industrial refrigeration, ASHRAE Journal 49 (8) (2007) 22-29.

[38] Simone, Hypervolume approximation (2015).

URL https://uk.mathworks.com/matlabcentral/fileexchange/ 50517-hypervolume-approximation 